\title{
Identification of phenolic constituents in Lonicera caerulea L. by HPLC with diode array detection electrospray ionisation tandem mass spectrometry
}

\author{
Mayya P. Razgonova ${ }^{1,2^{*}}$, Nadezhda G. Tikhonova ${ }^{1}$, Andrey S. Sabitov ${ }^{1}$, Natalia M. Mikhailova ${ }^{1}$, Svetlana R. Luchko ${ }^{1}$, \\ Alexander M. Zakharenko ${ }^{1,2}$, Konstantin S. Pikula ${ }^{1}$, and Kirill S. Golokhvast ${ }^{1,2,3,4}$ \\ ${ }^{1}$ N.I. Vavilov All-Russian Institute of Plant Genetic Resources, B. Morskaya 42-44, 190000, Saint-Petersburg, Russian Federation \\ ${ }^{2}$ Far Eastern Federal University, Sukhanova 8, 690950, Vladivostok, Russian Federation \\ ${ }^{3}$ Siberian Federal Scientific Centre of Agrobiotechnology, 633501, Centralnaya, Presidium, Krasnoobsk, Russian Federation \\ ${ }^{4}$ Pacific Geographical Institute, Far Eastern Branch of the Russian Academy of Sciences, Radio 7, 690041, Vladivostok, Russian \\ Federation
}

\begin{abstract}
The purpose of this work was a comparative metabolomic study of extracts of Blueberried honeysuckle Lonicera caerulea L.: №1043-11 (St. Petersburg); №1043-08 (St. Petersburg) №863; (Japan); №860 (Wild Lonicera from Amur river) from the collection of N.I. Vavilov All-Russian Institute of Plant Genetic Resources. To identify target analytes in extracts HPLC was used in combination with a BRUKER DALTONIKS ion trap. The results showed the presence of 82 target analytes corresponding to family Caprifoliaceae. In addition to the reported metabolites, a number of metabolites were newly annotated in Lonicera caerulea L. There were flavonols: Dihydrokaempferol, Rhamnetin I, Rhamnetin II, Taxifolin-3$O$-glucoside, Mearnsetin-hexoside, Horridin; flavones: Chrysoeriol, Apigenin- $O$-pentoside, Chrysoeriol-7- $O$ glucoside; flavanone Naringenin; flavan-3-ols: Catechin, Epicatechin, Biochanin A-7-O-glucoside; essential amino acids: L-Pyroglutamic acid, Tyrosine; polypeptide 5-Oxo-L-propyl-L-isoleucine; sterols: Ergosterol, Fucosterol, Beta-Sitosterin; triterpenoids: Betunolic acid, Oleanoic acid; anabolic steroid Vebonol, indole sesquiterpene alkaloid Sespendole; iridoids: Monotropein, $p$-Coumaroyl monotropein, $p$-Coumaroyl monotropein hexoside; Myristoleic acid, etc.
\end{abstract}

\section{Introduction}

Blue-berried honeysuckle Lonicera caerulea L., family Caprifoliaceae is known as a natural source of food, beverages and nutraceuticals due to its rich chemical composition, enriched with nutrient and biologically active compounds. The increased focus on these berries is due to their phenolic composition, antioxidant activity, and potential health benefits. The high content of phenols in Lonicera caerulea L. is directly related to their biological activity. Popularity of phenolic compounds has grown in recent years as they are excellent antioxidants. Antioxidant intake has been shown to be effective in preventing cancer, cardiovascular disease, osteoporosis, obesity, diabetes, and other health problems [Dias et al., 2017]. The antioxidant properties of plant phenolic compounds are relevant in the field of nutrition (inhibition of lipid oxidation), physiology (protection against oxidative stress) and cosmetology. Phenolic compounds provide antioxidant activity through direct reduction of reactive oxygen species (ROS), inhibition of enzymes involved in oxidative stress, binding of metal ions responsible for ROS production, and stimulation of endogenous antioxidant defense systems [Hossain et al., 2016]. The quality and quantity of phenolic compounds in plants usually depends on the stage of growth, the parts of the plant used and the growing conditions in the environment [Bujor O.-C., 2016].

In this regard, the purpose of this work is the simultaneous assessment of phenolic compounds in the berries of Lonicera caerulea $\mathrm{L}$. of various species collected in different climaticgeographical zones of Russia. This study is a complete qualitative study of phenols and other compounds, leading to the identification of a large number of phenolic secondary metabolites isolated from Lonicera caerulea L. berries of various species.

\footnotetext{
* Corresponding author: razgonova.mp@dvfu.ru (M.R.)
} 


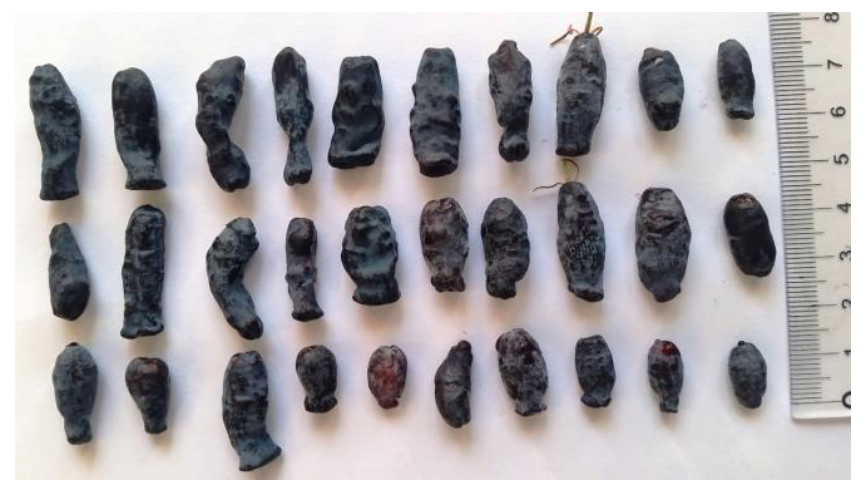

Fig. 1. Polymorphism of wild Lonicera caerulea L. berries presented in the collection of N.I. Vavilov All-Russian Institute of Plant Genetic Resources

Initial LC-MS/MS screening suggested that 82 target analytes detected in EtOH-extracts of Blue-berried honeysuckle. Therefore, tandem mass spectrometry was used in this study for comparative small molecule profiling of four Lonicera varieties cultivated in the collection of N.I. Vavilov All-Russian Institute of Plant Genetic Resources.

\section{Experimental}

\subsection{Materials}

The object of the study was the four varieties of Blue-berried honeysuckle Lonicera caerulea L. of breeding varieties obtained as a result of many years of research from the collection of N.I. Vavilov All-Russian Institute of Plant Genetic Resources. There were a varieties: №1043-11 (St. Petersburg); №1043-08 (St. Petersburg); №863 (Japan); №860 (Wild Lonicera from Amur river). The berries were harvested at the end of July 2020. All samples morphologically corresponded to the pharmacopoeial standards of the State Pharmacopoeia of the Russian Federation [SPh XIV, Russia, 2018].

\subsection{Chemicals and Reagents}

HPLC-grade acetonitrile was purchased from Fisher Scientific (Southborough, UK), MS-grade formic acid was from Sigma-Aldrich (Steinheim, Germany). Ultra-pure water was prepared from a SIEMENS ULTRA clear (SIEMENS water technologies, Germany), and all other chemicals were analytical grade.

\subsection{Fractional maceration.}

To obtain highly concentrated extracts, fractional maceration was applied. In this case, the total amount of the extractant (methyl alcohol of reagent grade) is divided into 3 parts and is consistently infused on potato with the first part, then with the second and third. The infusion time of each part of the extractant was 7 days.

\subsection{Liquid chromatography}

HPLC was performed using Shimadzu LC-20 Prominence HPLC (Shimadzu, Japan) was used, equipped with an UVsensor and a Shodex ODP-40 4E reverse phase column to perform the separation of multicomponent mixtures. The gradient elution program was as follows: $0.01-4 \mathrm{~min}, 100 \%$ $\mathrm{CH}_{3} \mathrm{CN}$; 4-35 min, $100-25 \% \mathrm{CH}_{3} \mathrm{CN} ; 35-50 \mathrm{~min}, 25-0 \%$ $\mathrm{CH}_{3} \mathrm{CN}$; control washing 50-60 min $0 \% \mathrm{CH}_{3} \mathrm{CN}$. The entire HPLC analysis was done with a ESI detector at wavelengths of $230 \mathrm{\eta m}$ and $330 \mathrm{\eta m}$; the temperature corresponded to $17^{\circ} \mathrm{C}$. The injection volume was $1 \mathrm{ml}$.

\subsection{Mass spectrometry}

MS analysis was performed on an ion trap amaZon SL (BRUKER DALTONIKS, Germany) equipped with an ESI source in negative ion mode. The optimized parameters were obtained as follows: ionization source temperature: $70^{\circ} \mathrm{C}$, gas flow: 41 / $\mathrm{min}$, nebulizer gas (atomizer): 7.3 psi, capillary voltage: $4500 \mathrm{~V}$, end plate bend voltage: $1500 \mathrm{~V}$, fragmentary: $280 \mathrm{~V}$, collision energy: $60 \mathrm{eV}$. An ion trap was used in the scan range m / z $100-1.700$ for MS and MS/MS. The capture rate was one spectrum/s for MS and two spectrum/s for MS/MS. Data collection was controlled by Windows software for BRUKER DALTONIKS. All experiments were repeated three times. A four-stage ion separation mode (MS/MS mode) was implemented.

\section{Results and discussion}

Four of the most consumed extracts of Lonicera caerulea L. have been selected. All of them have a rich bioactive composition. There were four extracts from a varieties: №1043-11 (St. Petersburg); №1043-08 (St. Petersburg); №863 (Japan); №860 (Wild Lonicera, Amur river) from the collection of N.I. Vavilov All-Russian Institute of Plant Genetic Resources.

High accuracy mass spectrometric data were recorded on an ion trap amaZon SL BRUKER DALTONIKS equipped with an ESI source in the mode of negative-positive ions. The four-stage ion separation mode (MS/MS mode) was implemented. The combination of both ionization modes (positive and negative) in MS full scan mode gave extra certainly to the molecular mass determination (Fig. 2,3,4). The positive-negative ion mode provides the highest sensitivity and results in limited fragmentation, making it most suited to infer the molecular mass of the separated polyphenols, especially in cases where concentration is low. By comparing the $\mathrm{m} / \mathrm{z}$ values, the RT and the fragmentation patterns with the $\mathrm{MS}^{2}$ spectral data taken from the literature [Abeywickrama et al., 2016; Abu-Reidah et al., 2015; Rafsanjany et al., 2015; Goufo et al., 2020; Paudel et al., 2013; Jaiswal et al., 2014; De Rosso et al., 2014; Marzouk et al., 2018; Barros et al., 2012; Pradhan \& Saha, 2016; da Silva et al., 2019; Ruiz et al., 2013; Ruiz et al., 2010; Razgonova et al., 2020; Kajdzanoska et al., 2010] or to search the data bases (MS2T, MassBank, HMDB). A unifying system table was compiled of the molecular masses of the target analytes isolated from the EtOH-extract of Lonicera caerulea L. for 
ease of identification (Table 1). The 82 target analytes shown in Table 1 belong to different polyphenolic families: flavones, flavonols, flavan-3-ols, flavanones, anthocyanins, hydroxycinnamic acids, hydroxybenzoic acids, stilbenes, proanthocyanidins and belong to others classes of compounds.

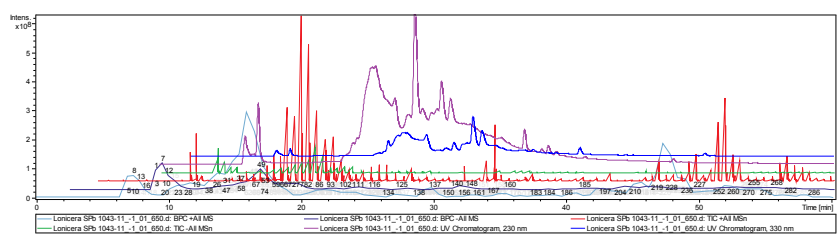

Fig.2. Chemical profiles of the Lonicera caerulea L. (variety SPb 1043-11) sample represented total ion chromatogram from $\mathrm{MeOH}-$ extract.

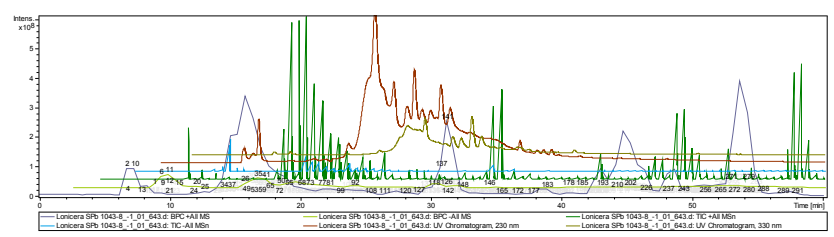

Fig.3. Chemical profiles of the Lonicera caerulea L. (variety $\mathrm{SPb} 1043-8$ ) sample represented total ion chromatogram from $\mathrm{MeOH}$-extract.

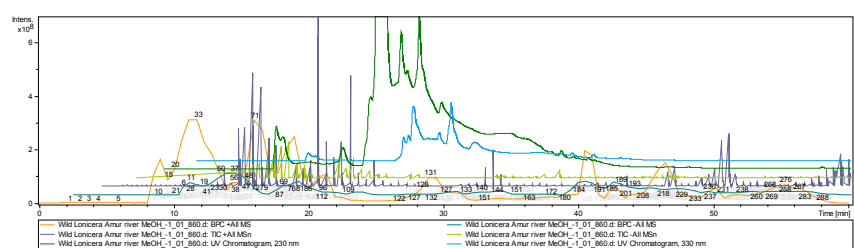

Fig.4. Chemical profiles of the Lonicera caerulea L. (variety Wild lonicera from Amur river) sample represented total ion chromatogram from $\mathrm{MeOH}$-extract.

In addition to the reported metabolites, a number of metabolites were newly annotated in Lonicera caerulea L. There were flavonols: Dihydrokaempferol, Rhamnetin I, Rhamnetin II, Taxifolin-3-O-glucoside, Mearnsetinhexoside, Horridin; flavones: Chrysoeriol, Apigenin-Opentoside, Chrysoeriol-7- $O$-glucoside; flavanone Naringenin; flavan-3-ols: Catechin, Epicatechin, Biochanin A-7-O-glucoside; essential amino acids: L-Pyroglutamic acid, Tyrosine; polypeptide 5-Oxo-L-propyl-L-isoleucine; sterols: Ergosterol, Fucosterol, Beta-Sitosterin; triterpenoids: Betunolic acid, Oleanoic acid; anabolic steroid Vebonol, indole sesquiterpene alkaloid Sespendole; iridoids: Monotropein, p-Coumaroyl monotropein, p-Coumaroyl monotropein hexoside; Myristoleic acid, etc. 
Table 1. Identified target analytes in $\mathrm{MeOH}$ extracts of berries of Lonicera caerulea $\mathrm{L}$.

\begin{tabular}{|c|c|c|c|c|c|c|c|c|c|c|}
\hline № & $\begin{array}{l}\text { № of } \\
\text { collection of } \\
\text { N.I. Vavilov } \\
\text { All-Russian } \\
\text { Institute of } \\
\text { Plant Genetic } \\
\text { Resources } \\
\end{array}$ & $\begin{array}{l}\text { Class of } \\
\text { compounds }\end{array}$ & Identification & Formula & $\begin{array}{l}\text { Calculate } \\
\text { d mass }\end{array}$ & $\begin{array}{l}\text { Observe } \\
\text { d mass } \\
{[\mathrm{M}-\mathrm{H}]-}\end{array}$ & $\begin{array}{c}\text { Obser- } \\
\text { ved } \\
\text { mass } \\
{[\mathbf{M}+\mathbf{H}]} \\
+\end{array}$ & $\begin{array}{c}\text { MS/MS } \\
\text { Stage 1 } \\
\text { fragment } \\
\text { a-tion }\end{array}$ & $\begin{array}{c}\text { MS/MS } \\
\text { Stage 2 } \\
\text { fragment } \\
\text { a-tion }\end{array}$ & $\begin{array}{c}\text { MS/MS } \\
\text { Stage 3 } \\
\text { fragment } \\
\text { a-tion }\end{array}$ \\
\hline & & POLYPHENOLS & & & & & & & & \\
\hline 1 & $\begin{array}{l}\text { SPb 1043-11; } \\
\text { SPb 1043-8 }\end{array}$ & Flavonol & Kaempferol & $\mathrm{C}_{15} \mathrm{H}_{10} \mathrm{O}_{6}$ & 286.2363 & & 287 & $269 ; 149$ & $239 ; 181$ & \\
\hline 2 & $\begin{array}{l}\text { 863; SPb } \\
1043-8\end{array}$ & Flavonol & Dihydrokaempferol & $\mathrm{C}_{15} \mathrm{H}_{12} \mathrm{O}_{6}$ & 288.2522 & & 289 & $\begin{array}{l}176 ; 144 ; \\
272\end{array}$ & 144 & 116 \\
\hline 3 & $\begin{array}{l}863 ; 860 ; \mathrm{SPb} \\
1043-8\end{array}$ & Flavonol & Quercetin & $\mathbf{C}_{15} \mathbf{H}_{10} \mathbf{O}_{7}$ & 302.2357 & & 303 & $257 ; 146$ & 229 & $201 ; 145$ \\
\hline 4 & SPb 1043-11 & Flavonol & $\begin{array}{l}\text { Rhamnetin I [beta-Rhamnocitrin; } \\
\text { Quercetin 7-Methyl ether] }\end{array}$ & $\mathrm{C}_{16} \mathrm{H}_{12} \mathrm{O}_{7}$ & 316.2623 & & 317 & $299 ; 213$ & 267 & 239 \\
\hline 5 & 863 & Flavonol & Rhamnetin II & $\mathrm{C}_{16} \mathrm{H}_{12} \mathrm{O}_{7}$ & 316.2623 & & 317 & 302 & $\begin{array}{c}274 ; 153 ; \\
121\end{array}$ & $\begin{array}{c}229 ; 153 \\
121\end{array}$ \\
\hline 6 & $\begin{array}{l}\text { 863; SPb } \\
1043-11 \\
\end{array}$ & Flavonol & $\begin{array}{l}\text { Isorhamnetin [Isorhamnetol; Quercetin 3'- } \\
\text { Methyl ether; 3-Methylquercetin] }\end{array}$ & $\mathrm{C}_{16} \mathrm{H}_{12} \mathrm{O}_{7}$ & 316.2623 & 315 & & 283 & $255 ; 211$ & 227 \\
\hline 7 & SPb 1043-8 & Flavonol & Kaempferol-3- $O$-hexoside & $\mathrm{C}_{21} \mathrm{H}_{20} \mathrm{O}_{11}$ & 448.3769 & & 449 & 287 & 213 & 213 \\
\hline 8 & 863 & Flavonol & Quercetin-3-(3-O-arabinosyl)glucoside & $\mathrm{C}_{26} \mathrm{H}_{28} \mathrm{O}_{16}$ & 596.4909 & & 597 & $303 ; 465$ & $257 ; 165$ & 229 \\
\hline 9 & $863 ; 860$ & Flavonol & $\begin{array}{l}\text { Quercetin 3-O-glucoside [ Isoquercitrin; } \\
\text { Hirsutrin; Quercetin-3-O-Glucopyranoside; } \\
\text { 3-Glucosylquercetin] }\end{array}$ & $\mathrm{C}_{21} \mathrm{H}_{20} \mathrm{O}_{12}$ & 464.3763 & & 465 & 303 & $229 ; 165$ & $201 ; 161$ \\
\hline 10 & $\begin{array}{l}863 ; \mathrm{SPb} \\
1043-11 ; \mathrm{SPb} \\
1043-8 ; 860 \\
\end{array}$ & Flavonol & Taxifolin-3-O-glucoside & $\mathrm{C}_{21} \mathrm{H}_{22} \mathrm{O}_{12}$ & 466.3922 & & 467 & $449 ; 287$ & $377 ; 279$ & $345 ; 283$ \\
\hline 11 & 860 & Flavonol & Kaempferol acetyl hexoside & $\mathrm{C}_{23} \mathrm{H}_{22} \mathrm{O}_{12}$ & 490.4136 & & 491 & 257 & 183 & \\
\hline 12 & $\mathrm{SPb}$ 1043-11 & Flavonol & Mearnsetin-hexoside & $\mathrm{C}_{22} \mathrm{H}_{22} \mathrm{O}_{13}$ & 494.4023 & & 495 & $477 ; 387$ & $\begin{array}{c}387 ; 315 \\
199\end{array}$ & \\
\hline 13 & 863 & Flavonol & $\begin{array}{l}\text { Horridin [Quercetin 3-Rhamnosyl-(1->2)- } \\
\text { Rhamnoside] }\end{array}$ & $\mathrm{C}_{27} \mathrm{H}_{30} \mathrm{O}_{15}$ & 594.5181 & & 595 & $\begin{array}{l}463 ; 432 \\
301\end{array}$ & 301 & 286 \\
\hline 14 & SPb 1043-11 & Flavonol & $\begin{array}{l}\text { Kaempferol 3-O-(6-O-rhamnosyl- } \\
\text { glucoside) }\end{array}$ & $\mathrm{C}_{27} \mathrm{H}_{30} \mathrm{O}_{15}$ & 594.5181 & & 595 & 287 & 213 & 185 \\
\hline
\end{tabular}




\begin{tabular}{|c|c|c|c|c|c|c|c|c|c|}
\hline 15 & $\begin{array}{l}\text { 863; SPb } \\
\text { 1043-11 }\end{array}$ & Flavonol & Rutin (Quercetin 3-O-rutinoside) & $\mathrm{C}_{27} \mathrm{H}_{30} \mathrm{O}_{16}$ & 610.5175 & 611 & $303 ; 197$ & $\begin{array}{l}285 ; 229 \\
195\end{array}$ & 229 \\
\hline 16 & 860 & Flavan-3-ol & Catechin [D-Catechol] & $\mathrm{C}_{15} \mathrm{H}_{14} \mathrm{O}_{6}$ & 290.2681 & 291 & $289 ; 159$ & $230 ; 127$ & \\
\hline 17 & SPb 1043-11 & Flavan-3-ol & Epicatechin & $\mathrm{C}_{15} \mathrm{H}_{14} \mathrm{O}_{6}$ & 290.2681 & 291 & $273 ; 137$ & & \\
\hline 18 & 863 & Flavan-3-ol & Biochanin A-7-O-glucoside & $\mathrm{C}_{22} \mathrm{H}_{22} \mathrm{O}_{10}$ & 446.4041 & 447 & $245 ; 187$ & 217 & $148 ; 182$ \\
\hline 19 & $\begin{array}{l}\text { SPb 1043-11; } \\
\text { SPb 1043-8 }\end{array}$ & Flavone & $\begin{array}{l}\text { Apigenin [5,7-Dixydroxy-2- } \\
\text { (40Hydroxyphenyl)-4H-Chromen-4-One] }\end{array}$ & $\mathrm{C}_{15} \mathrm{H}_{10} \mathrm{O}_{5}$ & 270.2369 & 271 & 225 & 179 & \\
\hline 20 & SPb 1043-11 & Flavone & Chrysoeriol [Chryseriol] & $\mathrm{C}_{16} \mathrm{H}_{12} \mathrm{O}_{6}$ & 300.2629 & 301 & $255 ; 157$ & 209 & 135 \\
\hline 21 & SPb 1043-11 & Flavone & Apigenin-O-pentoside & & 448 & 449 & $\begin{array}{l}403 ; 287 ; \\
216\end{array}$ & $347 ; 137$ & 291 \\
\hline 22 & $\begin{array}{l}\text { 863; 860; SPb } \\
\text { 1043-11 }\end{array}$ & Flavone & Luteolin 7-O-glucoside [Cynaroside] & $\mathrm{C}_{21} \mathrm{H}_{20} \mathrm{O}_{11}$ & 448.3769 & 449 & 287 & $\begin{array}{l}269 ; 241 ; \\
132\end{array}$ & 133 \\
\hline 23 & 860 & Flavone & Chrysoeriol-7-O-glucoside & $\mathrm{C}_{22} \mathrm{H}_{22} \mathrm{O}_{11}$ & 462.4035 & 463 & $301 ; 243$ & 183 & \\
\hline 24 & SPb 1043-11 & Flavone & $\begin{array}{l}\text { Diosmin [Diosmetin-7-O-rutinoside; } \\
\text { Barosmin; Diosimin] }\end{array}$ & $\mathrm{C}_{28} \mathrm{H}_{32} \mathrm{O}_{15}$ & 608.5447 & 609 & $591 ; 531$ & 531 & 487 \\
\hline 25 & 863 & Flavanone & Naringenin [Naringetol; Naringenine] & $\mathrm{C}_{15} \mathrm{H}_{12} \mathrm{O}_{5}$ & 272.5228 & 273 & $147 ; 246$ & & \\
\hline 26 & SPb 1043-11 & Anthocyanin & Delphinidin & $\mathrm{C}_{15} \mathrm{H}_{11} \mathrm{O}_{7}$ & 303.2436 & 304 & $212 ; 149$ & $212 ; 145$ & \\
\hline 27 & SPb 1043-11 & Anthocyanin & Petunidin & $\mathbf{C}_{16} \mathbf{H}_{13} \mathbf{O}_{7+}$ & 317.2702 & 318 & 256 & $238 ; 113$ & 238 \\
\hline 28 & 863 & Anthocyanin & Cyanidin-pentoside & $\mathrm{C}_{20} \mathrm{H}_{19} \mathrm{O}_{10}$ & 419.3589 & 419 & 287 & $\begin{array}{c}259 ; 188 \\
133\end{array}$ & 160 \\
\hline 29 & $\begin{array}{l}\text { 863; SPb } \\
1043-11\end{array}$ & Anthocyanin & $\begin{array}{l}\text { Cyanidin-3-O-glucoside [Cyanidin 3-O- } \\
\text { beta-D-Glucoside] }\end{array}$ & $\begin{array}{c}\mathrm{C}_{21} \mathrm{H}_{21} \mathrm{O}_{11} \\
+\end{array}$ & 449.3848 & 449 & 287 & 287; 213; & $185 ; 141$ \\
\hline 30 & SPb 1043-11 & Anthocyanin & Peonidin-3-O-galactoside & $\begin{array}{c}\mathrm{C}_{22} \mathrm{H}_{23} \mathrm{O}_{11} \\
+\end{array}$ & 463.4114 & 463 & 301 & 286 & $258 ; 150$ \\
\hline 31 & SPb 1043-8 & Anthocyanin & Peonidin-3-O-glucoside & $\begin{array}{c}\mathrm{C}_{22} \mathrm{H}_{23} \mathrm{O}_{11} \\
+ \\
\end{array}$ & 463.4114 & 463 & 301 & 286 & $258 ; 200$ \\
\hline 32 & 863 & Anthocyanin & Peonidin 3-O-acetyl hexoside & $\mathrm{C}_{24} \mathrm{H}_{25} \mathrm{O}_{12}$ & 505.4481 & 506 & $303 ; 487$ & $\begin{array}{l}303 ; 229 \\
165\end{array}$ & $201 ; 159$ \\
\hline 33 & $\begin{array}{l}\text { SPb 1043-11; } \\
\text { SPb 1043-8; }\end{array}$ & Anthocyanin & Delphinidin 3-O-Beta- $D$-sambubioside & $\mathrm{C}_{26} \mathrm{H}_{29} \mathrm{O}_{16}$ & 597.4989 & 597 & $\begin{array}{l}303 ; 465 ; \\
229\end{array}$ & $229 ; 165$ & $201 ; 172$ \\
\hline 34 & $\begin{array}{l}\text { SPb 1043-11; } \\
\text { SPb 1043-8; }\end{array}$ & Anthocyanin & Peonidin 3-O-rutinoside & $\mathrm{C}_{28} \mathrm{H}_{33} \mathrm{O}_{15}$ & 609.5526 & 609 & $301 ; 463$ & 286 & 258 \\
\hline 35 & $\begin{array}{l}863 ; 860 ; \text { SPb } \\
1043-11 ; \text { SPb } \\
1043-8\end{array}$ & Anthocyanin & Cyanidin 3,5-O-diglucoside & $\mathrm{C}_{27} \mathrm{H}_{31} \mathrm{O}_{16}$ & 611.5335 & 611 & $287 ; 449$ & $287 ; 213$ & 185 \\
\hline
\end{tabular}




\begin{tabular}{|c|c|c|c|c|c|c|c|c|c|c|}
\hline 36 & SPb 1043-11 & Anthocyanin & $\begin{array}{l}\text { Peonidin-3,5- } \boldsymbol{O} \text {-diglucoside [Peonin; } \\
\text { Peonidin 3-Glucoside-5-Glucoside] }\end{array}$ & $\mathrm{C}_{28} \mathrm{H}_{33} \mathrm{O}_{16}$ & 625.5520 & & 625 & $301 ; 463$ & 286 & 258 \\
\hline 37 & 863 & $\begin{array}{c}\text { Hydroxybenzoic } \\
\text { acid (Phenolic } \\
\text { acid) } \\
\end{array}$ & $\begin{array}{l}\text { 4-Hydroxybenzoic acid [PHBA; Benzoic } \\
\text { acid; p-Hydroxybenzoic acid] }\end{array}$ & $\mathrm{C}_{7} \mathrm{H}_{6} \mathrm{O}_{3}$ & 138.1207 & & 139 & 121 & & \\
\hline 38 & $863 ; 860$ & $\begin{array}{c}\text { Hydroxybenzoic } \\
\text { acid (Phenolic } \\
\text { acid) }\end{array}$ & Gallic acid & $\mathrm{C}_{7} \mathrm{H}_{6} \mathrm{O}_{5}$ & 170.1195 & & 171 & 139 & 111 & \\
\hline 39 & $\begin{array}{l}863 ; \mathrm{SPb} \\
1043-11\end{array}$ & $\begin{array}{l}\text { Hydroxybenzoic } \\
\text { acid (Phenolic } \\
\text { acid) }\end{array}$ & $\begin{array}{l}\text { Ellagic acid [Benzoaric acid; Elagostasine; } \\
\text { Lagistase; Eleagic acid] }\end{array}$ & $\mathrm{C}_{14} \mathrm{H}_{6} \mathrm{O}_{8}$ & 302.1926 & 301 & & 257 & 229 & 201 \\
\hline 40 & 863 & $\begin{array}{l}\text { Hydroxybenzoic } \\
\text { acid (Phenolic } \\
\text { acid) }\end{array}$ & Salvianolic acid D & $\mathrm{C}_{20} \mathrm{H}_{18} \mathrm{O}_{10}$ & 418.3509 & 417 & & 373 & 347 & 303 \\
\hline 41 & $\begin{array}{l}\text { SPb 1043-11; } \\
\text { SPb 1043-8 }\end{array}$ & $\begin{array}{l}\text { Methylbenzoic } \\
\text { acid }\end{array}$ & Methylgallic acid [Methyl gallate] & $\mathrm{C}_{8} \mathrm{H}_{8} \mathrm{O}_{5}$ & 184.1461 & & 185 & 139 & 111 & \\
\hline 42 & 863 & $\begin{array}{l}\text { Hydroxycinnamic } \\
\text { acid }\end{array}$ & $\begin{array}{l}\text { Cinnamic acid (Trans-cinnamic acid; } \\
\text { Phenylacrylic acid) }\end{array}$ & $\mathrm{C}_{9} \mathrm{H}_{8} \mathrm{O}_{2}$ & 148.1586 & 147 & & 129 & & \\
\hline 43 & SPb 1043-11 & $\begin{array}{l}\text { Hydroxycinnamic } \\
\text { acid }\end{array}$ & $\begin{array}{l}\text { Caffeic acid [(2E)-3-(3,4- } \\
\text { Dihydroxyphenyl)acrylic acid] }\end{array}$ & $\mathrm{C}_{9} \mathrm{H}_{8} \mathrm{O}_{4}$ & 180.1574 & & 181 & 135 & 119 & \\
\hline 44 & $\begin{array}{l}\text { 863; 860; SPb } \\
1043-11 ; \text { SPb } \\
1043-8\end{array}$ & $\begin{array}{l}\text { Hydroxycinnamic } \\
\text { acid }\end{array}$ & $\begin{array}{l}\text { Chlorogenic acid [3-O-Caffeoylquinic } \\
\text { acid] }\end{array}$ & $\mathrm{C}_{16} \mathrm{H}_{18} \mathrm{O}_{9}$ & 354.3087 & 353 & & 191 & 127 & \\
\hline 45 & 860 & $\begin{array}{l}\text { Hydroxycinnamic } \\
\text { acid }\end{array}$ & Feruloylquinic acid & $\mathrm{C}_{17} \mathrm{H}_{20} \mathrm{O}_{9}$ & 368.3353 & 367 & & $179 ; 135$ & 135 & \\
\hline 46 & 863 & $\begin{array}{l}\text { Hydroxycinnamic } \\
\text { acid }\end{array}$ & 5-O-Feruloylquinic acid & $\mathrm{C}_{17} \mathrm{H}_{20} \mathrm{O}_{9}$ & 368.3353 & & 369 & 163 & 145 & 117 \\
\hline 47 & $\begin{array}{l}\text { SPb 1043-11; } \\
\text { SPb 1043-8 }\end{array}$ & $\begin{array}{l}\text { Hydroxycinnamic } \\
\text { acid }\end{array}$ & 3-O-Hydroxydihydrocaffeoylquinic acid & $\mathrm{C}_{16} \mathrm{H}_{20} \mathrm{O}_{10}$ & 372.3240 & 371 & & 191 & 127 & \\
\hline 48 & $863 ; 860$; & $\begin{array}{l}\text { Hydroxycinnamic } \\
\text { acid }\end{array}$ & 5-O-(4'-O-Feruloyl glucosyl)quinic acid & $\mathrm{C}_{23} \mathrm{H}_{30} \mathrm{O}_{14}$ & 530.4759 & & 531 & $299 ; 245$ & $281 ; 167$ & 149 \\
\hline 49 & $\begin{array}{l}\text { 863; SPb } \\
1043-11\end{array}$ & Cinnamic acid & Ferulic acid & $\mathrm{C}_{10} \mathrm{H}_{10} \mathrm{O}_{4}$ & 194.184 & 193 & & 161 & 133 & \\
\hline 50 & 860 & Stilbene & $\begin{array}{l}\text { Resveratrol [trans-Resveratrol; 3,4',5- } \\
\text { Trihydroxystilbene; Stilbentriol] }\end{array}$ & $\mathrm{C}_{14} \mathrm{H}_{12} \mathrm{O}_{3}$ & 228.2433 & & 229 & 211 & $183 ; 127$ & 138 \\
\hline 51 & 863 & Stilbene & Oxyresveratrol & $\mathrm{C}_{14} \mathrm{H}_{12} \mathrm{O}_{4}$ & 244.2427 & & 245 & $\begin{array}{l}220 ; 173 \\
112\end{array}$ & & \\
\hline 52 & 860 & $\begin{array}{c}\text { Oligomeric } \\
\text { proanthocyanidins }\end{array}$ & Epiafzelechin [(epi)Afzelechin] & $\mathrm{C}_{15} \mathrm{H}_{14} \mathrm{O}_{5}$ & 274.2687 & & 275 & $185 ; 244$ & 157 & \\
\hline
\end{tabular}




\begin{tabular}{|c|c|c|c|c|c|c|c|c|c|c|}
\hline 53 & SPb 1043-11 & $\begin{array}{c}\text { Oligomeric } \\
\text { proanthocyanidins }\end{array}$ & (Epi)Catechin-A-(epi)afzelechin & $\mathrm{C}_{30} \mathrm{H}_{24} \mathrm{O}_{11}$ & 560.5050 & & 561 & $\begin{array}{l}399 ; 278 \\
201\end{array}$ & $\begin{array}{l}325 ; 255 \\
191 ; 132\end{array}$ & \\
\hline 54 & $\mathrm{SPb}$ 1043-11 & $\begin{array}{c}\text { Oligomeric } \\
\text { proanthocyanidins }\end{array}$ & (Epi)catechin-(4,8'/2,6')-(epi)catechin & & 576 & & 577 & $\begin{array}{l}559 ; 447 ; \\
377 ; 306 ; \\
265 ; 179 \\
\end{array}$ & $247 ; 121$ & 175 \\
\hline 55 & SPb 1043-11 & $\begin{array}{c}\text { Oligomeric } \\
\text { proanthocyanidin }\end{array}$ & $\begin{array}{l}\text { 3-O-Galloyl (epi)catechin-(4,8)- } \\
\text { (epi)gallocatechin }\end{array}$ & & 746 & & 748 & $\begin{array}{l}575 ; 466 ; \\
379 ; 270\end{array}$ & $\begin{array}{l}318 ; 264 \\
175\end{array}$ & $235 ; 161$ \\
\hline \multirow[t]{2}{*}{56} & $\begin{array}{l}\text { SPb 1043-11; } \\
\text { SPb 1043-8 }\end{array}$ & $\begin{array}{l}\text { Aryl-beta- } \\
\text { glycoside }\end{array}$ & Arbutin & $\mathbf{C}_{12} \mathbf{H}_{16} \mathrm{O}_{7}$ & 272.2512 & & 273 & 227 & 181 & 135 \\
\hline & & OTHERS & & & & & & & & \\
\hline 57 & SPb 1043-8 & $\begin{array}{c}\text { Non-proteinogenic } \\
\text { L-alpha-amino } \\
\text { acid }\end{array}$ & $\begin{array}{l}\text { L-Pyroglutamic acid [Pidolic acid; 5- } \\
\text { Oxo-L-Proline] }\end{array}$ & $\mathrm{C}_{5} \mathrm{H}_{7} \mathrm{NO}_{3}$ & 129.1140 & & 130 & 111 & & \\
\hline 58 & $\mathrm{SPb}$ 1043-11 & Amino acid & $\begin{array}{l}\text { Leucine [(S)-2-Amino-Methylpentanoic } \\
\text { acid] }\end{array}$ & $\mathrm{C}_{6} \mathrm{H}_{13} \mathrm{NO}_{2}$ & 131.1729 & & 132 & 130 & 112 & \\
\hline 59 & $\begin{array}{l}\text { 860; SPb } \\
1043-8\end{array}$ & Amino acid & Phenylalanine [L-Phenylalanine] & $\mathrm{C}_{9} \mathrm{H}_{11} \mathrm{NO}_{2}$ & 165.1891 & & 166 & 120 & & \\
\hline 60 & $\begin{array}{l}\text { SPb 1043-11; } \\
\text { SPb 1043-8; } \\
860\end{array}$ & $\begin{array}{l}\text { Cyclohexenecarbo } \\
\text { xylic acid }\end{array}$ & Shikimic acid [L-Schikimic acid] & $\mathrm{C}_{7} \mathrm{H}_{10} \mathrm{O}_{5}$ & 174.1513 & & 175 & 128 & 111 & \\
\hline 61 & 863 & Amino acid & $\begin{array}{l}\text { Tyrosine [(2S)-2-Amino-3-(4- } \\
\text { Hydroxyphnyl)Propanoic acid] }\end{array}$ & $\mathrm{C}_{9} \mathrm{H}_{11} \mathrm{NO}_{3}$ & 181.1885 & & 179 & 133 & 115 & \\
\hline 62 & SPb 1043-8 & Propenyl & Methyl eugenol & $\mathrm{C}_{11} \mathrm{H}_{14} \mathrm{O}_{2}$ & 178.2277 & & 179 & 151 & 123 & \\
\hline 63 & 863 & Dicarboxylic acid & $\begin{array}{l}\text { Azelaic acid [Nonanedioic acid; Anchoic } \\
\text { acid; Finacea] }\end{array}$ & $\mathrm{C}_{9} \mathrm{H}_{16} \mathrm{O}_{4}$ & 188.2209 & & 189 & 171 & 139 & 111 \\
\hline 64 & $\begin{array}{l}863 ; 860 ; \mathrm{SPb} \\
1043-8\end{array}$ & Tricarboxylic acid & Citric acid [ Anhydrous; Citrate] & $\mathrm{C}_{6} \mathrm{H}_{8} \mathrm{O}_{7}$ & 192.1235 & 191 & & $111 ; 173$ & & \\
\hline 65 & SPb 1043-8 & $\begin{array}{c}\text { Polyhydroxycarbo } \\
\text { xylic acid }\end{array}$ & Quinic acid & $\mathrm{C}_{7} \mathrm{H}_{12} \mathrm{O}_{6}$ & 192.1666 & 191 & & $111 ; 173$ & 111 & \\
\hline 66 & 863 & Propanoic acid & Dihydroferulic acid & $\mathrm{C}_{10} \mathrm{H}_{12} \mathrm{O}_{4}$ & 196.1999 & & 197 & 127 & & \\
\hline 67 & $\begin{array}{l}\text { 863; SPb } \\
1043-8 \\
\end{array}$ & Carboxylic acid & $\begin{array}{l}\text { Myristoleic acid [Cis-9-Tetradecanoic } \\
\text { acid] }\end{array}$ & $\mathrm{C}_{14} \mathrm{H}_{26} \mathrm{O}_{2}$ & 226.3550 & & 227 & $209 ; 165$ & 121 & \\
\hline 68 & $\begin{array}{l}\text { SPb 1043-11; } \\
\text { SPb 1043-8 }\end{array}$ & Polypeptide & 5-Oxo-L-propyl-L-isoleucine & $\begin{array}{c}\mathrm{C}_{11} \mathrm{H}_{18 N_{2}} \\
\mathrm{O}_{4}\end{array}$ & 242.2716 & & 243 & $196 ; 137$ & 151 & \\
\hline 69 & 863 & $\begin{array}{l}\text { Medium-chain } \\
\text { fatty acid }\end{array}$ & Hydroxy dodecanoic acid & $\mathrm{C}_{12} \mathrm{H}_{22} \mathrm{O}_{5}$ & 246.3001 & & 247 & 229 & 187 & \\
\hline
\end{tabular}




\begin{tabular}{|c|c|c|c|c|c|c|c|c|c|c|}
\hline 70 & 863 & $\begin{array}{l}\text { Terpenoid } \\
\text { trilactone }\end{array}$ & Bilobalide [ (-)-Bilobalide] & $\mathrm{C}_{15} \mathrm{H}_{18} \mathrm{O}_{8}$ & 326.2986 & 325 & & 183; & 119 & \\
\hline 71 & 860 & Iridoid & Monotropein & $\mathrm{C}_{16} \mathrm{H}_{22} \mathrm{O}_{11}$ & 390.3393 & & 391 & $219 ; 372$ & $202 ; 148$ & 160 \\
\hline 72 & 863 & Phytosterol & Ergosterol [Provitamin D2; Ergosterin] & $\mathrm{C}_{28} \mathrm{H}_{44} \mathrm{O}$ & 396.6484 & & 397 & $\begin{array}{l}379 ; 291 \\
206\end{array}$ & $291 ; 223$ & 139 \\
\hline 73 & 860 & Sterol & $\begin{array}{l}\text { Fucosterol [Fucostein; Trans-24- } \\
\text { Ethylidenecholesterol] }\end{array}$ & $\mathrm{C}_{29} \mathrm{H}_{48} \mathrm{O}$ & 412.6908 & & 413 & $\begin{array}{l}395 ; 324 \\
219\end{array}$ & 329 & \\
\hline 74 & $\begin{array}{l}863 ; \mathrm{SPb} \\
1043-11\end{array}$ & Sterol & Beta-Sitosterin [Beta-Sitosterol] & $\mathrm{C}_{29} \mathrm{H}_{50} \mathrm{O}$ & 414.7067 & & 415 & $216 ; 312$ & $159 ; 115$ & \\
\hline 75 & SPb 1043-11 & Anabolic steroid & Vebonol & $\mathrm{C}_{30} \mathrm{H}_{44} \mathrm{O}_{3}$ & 452.6686 & & 453 & 435 & $336 ; 226$ & $209 ; 139$ \\
\hline 76 & 860 & Triterpenoid & Betunolic acid & $\mathrm{C}_{30} \mathrm{H}_{46} \mathrm{O}_{3}$ & 454.6844 & & 455 & $\begin{array}{l}437 ; 345 ; \\
247\end{array}$ & $326 ; 283$ & $\begin{array}{l}303 ; 239 \\
\quad 199\end{array}$ \\
\hline 77 & 863 & Triterpenic acid & Oleanoic acid & $\mathrm{C}_{30} \mathrm{H}_{48} \mathrm{O}_{3}$ & 456.7003 & & 457 & $\begin{array}{l}425 ; 295 \\
225\end{array}$ & 167 & \\
\hline 78 & $\begin{array}{l}863 ; \mathrm{SPb} \\
1043-11\end{array}$ & $\begin{array}{l}\text { Thromboxane } \\
\text { receptor } \\
\text { antagonist }\end{array}$ & Vapiprost & $\begin{array}{c}\mathrm{C}_{30} \mathrm{H}_{39} \mathrm{NO} \\
4\end{array}$ & 477.6350 & & 478 & 337 & $263 ; 121$ & 119 \\
\hline 79 & $\begin{array}{l}\text { 863; SPb } \\
1043-11\end{array}$ & $\begin{array}{c}\text { Indole } \\
\text { sesquiterpene } \\
\text { alkaloid }\end{array}$ & Sespendole & $\begin{array}{c}\mathrm{C}_{33} \mathrm{H}_{45} \mathrm{NO} \\
4\end{array}$ & 519.7147 & & 520 & 184 & 125 & \\
\hline 80 & 863 & Iridoid glucoside & $p$-Coumaroyl monotropein & $\mathrm{C}_{25} \mathrm{H}_{28} \mathrm{O}_{13}$ & 536.4820 & & 537 & $\begin{array}{l}375 ; 256 \\
\quad 185\end{array}$ & & \\
\hline 81 & $\begin{array}{l}\text { 863; SPb } \\
1043-11\end{array}$ & Iridoid & p-Coumaroyl monotropein hexoside & & 698.8810 & & 699 & $\begin{array}{l}537 ; 347 \\
259\end{array}$ & $\begin{array}{l}375 ; 259 \\
\quad 185\end{array}$ & \\
\hline 82 & SPb 1043-11 & Steroidal alkaloid & Alpha-chaconine & $\begin{array}{c}\mathrm{C}_{45} \mathrm{H}_{73} \mathrm{NO} \\
{ }_{14}\end{array}$ & 852.0594 & & 852 & 706 & $560 ; 398$ & $398 ; 204$ \\
\hline
\end{tabular}


The CID-spectrum (collision induced dissociation spectrum) in positive ion modes of Dihydrokaempferol from extracts of Lonicera caerulea L. (variety $\mathrm{SPb} 1043$ 8 ) is shown in Fig. 5. The $[\mathrm{M}+\mathrm{H}]^{+}$ion produced three fragment ions at $m / z$ 270.99, $m / z$ 193.01, $\mathrm{m} / z$. 127.03 (Fig.
5). It was identified in the bibliography in extracts from Potato [Oertel et al., 2017]; F. glaucescens [Hamed et al., 2020]; Echinops [Seukep et al., 2020]; Rhodiola rosea [Lee et al., 2016]; Rhodiola crenulata [Daikonya et al., 2011].

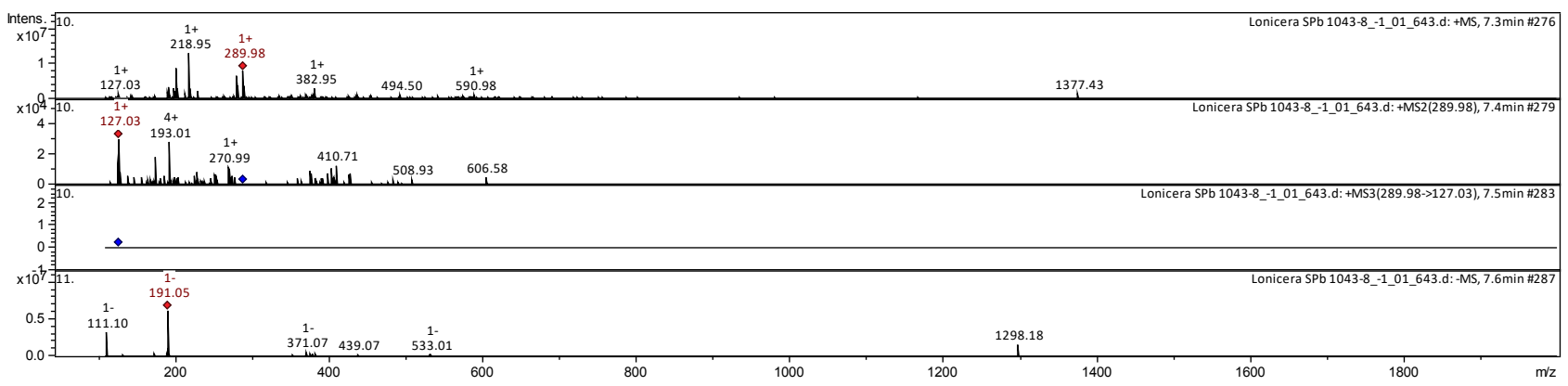

Fig.5. CID-spectrum of dihydrokaempferol from extracts of Lonicera caerulea $\mathrm{L}$. (variety SPb 1043-8), $m / z 289.98$.

The CID-spectrum in positive ion modes of Dihydrokaempferol from extracts of Lonicera caerulea $\mathrm{L}$. (variety Wild Lonicera from Amur river) is shown in Fig. 6. The $[\mathrm{M}+\mathrm{H}]^{+}$ion produced one fragment ion at $\mathrm{m} / \mathrm{z}$ 448.92 (Fig. 6). The fragment ion with $\mathrm{m} / z 448.92$ yields three daughter ions at $\mathrm{m} / \mathrm{z} 376.96, \mathrm{~m} / \mathrm{z} 344.93$, and $\mathrm{m} / \mathrm{z}$
286.95. The fragment ion with $\mathrm{m} / \mathrm{z} 376.96$ yields two daughter ions at $\mathrm{m} / \mathrm{z}, 344.92$, and $\mathrm{m} / \mathrm{z}$ 286.99. It was identified in the bibliography in extracts from Rubus ulmifolius [da Silva et al., 2019]; Vitis vinifera [Goufo et a., 2020]

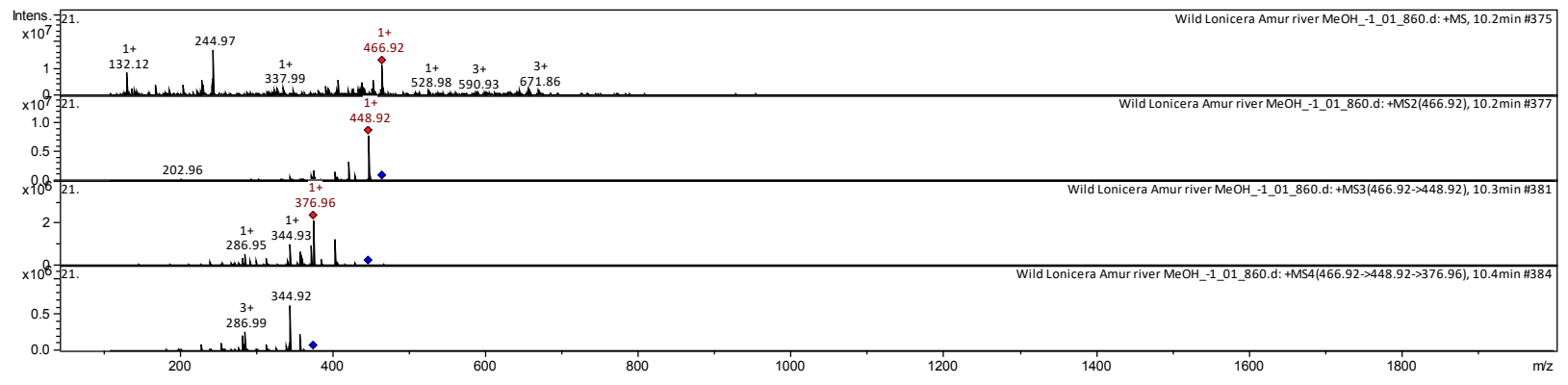

Fig.6. CID-spectrum of Taxifolin 3-O-glucoside from extracts of Lonicera caerulea L. (variety Wild Lonicera from Amur river), $m / z$ 466.92.

\section{Conclusions}

Blue-berried honeysuckle Lonicera caerulea L. contains a large number of polyphenolic compounds and other biologically active substances. In this work, we first tried to conduct a comparative metabolomic study of biologically active substances of wild Blue-berried honeysuckle obtained from locations in Khabarovsk territory and from the collection of N.I. Vavilov AllRussian Institute of Plant Genetic Resources (St.Petersburg). HPLC in combination with a BRUKER DALTONIKS ion trap (tandem mass spectrometry) was used to identify target analytes in extracts.

The results showed the presence of 82 biologically active compounds corresponding to the Blue-berried honeysuckle Lonicera caerulea species. In addition to the reported metabolites, a number of metabolites were newly annotated in blue-berried honeysuckle. There were flavonols: Dihydrokaempferol, Rhamnetin I, Rhamnetin II, Taxifolin-3-O-glucoside, Mearnsetin-hexoside, Horridin; flavones: Chrysoeriol, Apigenin- $O$-pentoside, Chrysoeriol-7- $O$-glucoside; flavanone Naringenin; flavan-3-ols: Catechin, Epicatechin, Biochanin A-7-Oglucoside; essential amino acids: L-Pyroglutamic acid,
Tyrosine; polypeptide 5-Oxo-L-propyl-L-isoleucine; sterols: Ergosterol, Fucosterol, Beta-Sitosterin; triterpenoids: Betunolic acid, Oleanoic acid; anabolic steroid Vebonol, indole sesquiterpene alkaloid Sespendole; iridoids: Monotropein, $p$-Coumaroyl monotropein, $p$-Coumaroyl monotropein hexoside; Myristoleic acid, etc.

The findings may support future research into the production of various pharmaceutical and dietary supplements containing blue-berried honeysuckle Lonicera caerulea L. extracts. A wide variety of biologically active compounds opens up rich opportunities for the creation of new drugs and biologically active additives based on extracts from this family Caprifoliaceae.

\section{References}

1. G. Abeywickram; S.C. Debnath, P. Ambigaipalan, F. Shahidi, J. Agric. Food Chem, 64(49) (2016)

2. I.M. Abu-Reidah, M. S. Ali-Shtayeh, R. M. Jamous, D. Arraes-Roman, A. Segura-Carretero, Food Chem, 166 179-191 (2015). 
3. L. Barros, M. Duenas, A. M. Carvalho, I.C.F.R. Ferreira, C. Santos-Buelga, Food and Chem, 50(5) 1576-1582 (2012)

4. O.-C. Bujor Extraction, identification and antioxidant activity of the phenolic secondary metabolites isolated from the leaves, stems and fruits of two shrubs of the Ericaceae family (PhD THESIS, 2016)

5. A. Daikonya, S. Kitanaka, J Food Chem Safety, 18(3) 183-190 (2011)

6. L.P. Da Silva, E. Pereira, T.C.S.P. Pires, M.J. Alves, O.R. Pereira, L. Barros I.C.F.R. Ferreira, Food Res. Int., 119 34-43 (2019)

7. M. De Rosso, L. Tonidandel, R. Larcher, G. Nicolini, A. Dalla Vedova, F. De Marchi, M. Gardiman, M. Giust, R. Flamini, Food Chem, 163 244-251 (2014)

8. T.R. Dias, M.G. Alves, S. Casal, P.F. Oliveira, B.M. Silva, Current Med. Chem., 24 334-354 (2017)

9. P. Goufo, R.K. Singh, I. Cortez, Antioxidants, 9398 (2020)

10. A.R. Hamed, S.S .El-Hawary, R.M. Ibrahim, U.R. Abdelmohsen, A.M. El-Halawany, J. Chrom. Sci., 19 (2020)

11. M.K. Hossain, A.A. Dayem, J. Han, Y. Yin, K. Kim, S.K. Saha, G.-M. Yang, H.Y.Choi, S.-G. Cho, Int. J. Mol. Sci., 17569 (2016)

12. R. Jaiswal, H. Muller, A. Muller, M.G.E. Karar, N. Kuhnert, Phytochem. 108 252-263 (2014)

13. M. Kajdzanoska, V. Gjamovski, M.Stefova, Macedonian J. of Chemistry and Chemical Engr., 29(2) 181-194 (2010)
14. T.H. Lee, C.C. Hsu, G. Hsiao.; J.Y. Fang, W.M. Liu, C.K. Lee, Planta Med., 82(8) 698-704 (2016)

15. M.M. Marzouk, S.R. Hussein, A. Elkhateeb, M. Elshabrawy, E.-S. S. Abdel-Hameed, S.A. Kawashty, J. Applied Pharm. Sci., 8(08) 116-122 (2018)

16. A. Oertel, A. Matros, A. Hartmann, P. Arapitsas K.J. Dehmer, S. Martens, H.P. Mock, Planta, 246 281-297 (2017)

17. P.C. Pradhan, S .Saha, J Food Sci. Technol., 53(2) 1205-1213 (2016)

18. L. Paudel, F.J. Wyzgovski, J.C. Scheerens, A.M. Chanon, R.N. Reese, D. Smiljanic, C. Wesdemiotis, J.J. Blakeslee, K.M. Riedl, P.L. Rinaldi, J. Agricult. Food. Chem., 61 12032-12043 (20130

19. N. Rafsanjany, J. Senker, S. Brandt, U. Dobrindt, A. Hensel, J. Agric. Food Chem., 63 8804-8818 (2015)

20. M.P. Razgonova, A.M. Zakharenko, V. Grudev, S. Ercisli, K.S. Golokhvast, Molecules 2537742020

21. A. Ruiz, I. Hermosin-Gutierrez, C. Mardones, C. Vergara, E. Herlitz, M. Vega, C. Dorau, P. Winterhalter, von D. Baer, Agric. \& Food Chem., 51 706-713 (2010)

22. A. Ruiz, I. Hermosin-Gutierrez, C. Vergara, D. von Baer, M. Zapata, A. Hitschfild, L. Obando, C. Mardones, Food Res. Int., 51 706-713 (2013)

23. A.J. Seukep, Y.-L. Zhang, Y.-B .Xu, M.-Q. Guo, Pharmaceut., 1359 (2020)

24. State Pharmacopeia XIV (in Russ., 2018) 